\title{
Managing a COVID 19 patient at different health care and field level settings
}

\section{Arti Gupta ${ }^{1}$, Rakesh Kakkar ${ }^{2}$}

${ }^{1}$ Assistant Professor, Department of Community and Family Medicine, All India Institute of Medical Sciences Mangalagiri, Andhra Pradesh, India; ${ }^{2}$ Professor and Head, Department of Community and Family Medicine, All India Institute of Medical Sciences Mangalagiri, Andhra Pradesh, India

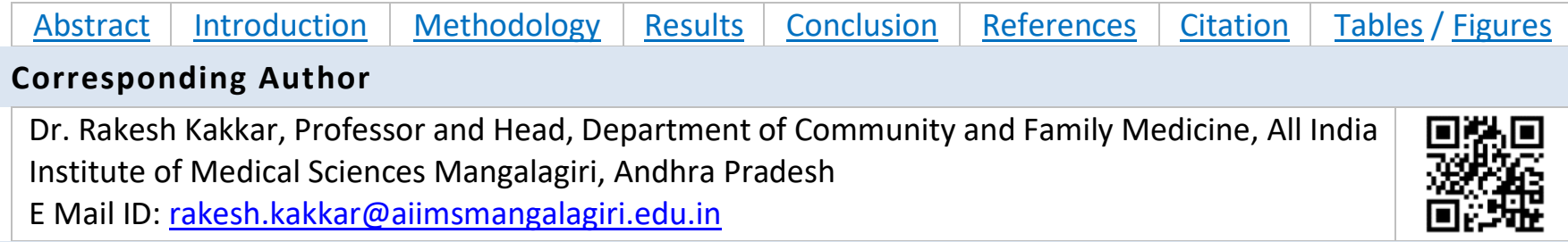

\section{Citation}

Gupta A, Kakkar R. Managing a COVID 19 patient at different health care and field level settings. Indian J Comm Health. 2020;32(2-Special Issue):188-195.

Source of Funding: Nil Conflict of Interest: None declared

\section{Article Cycle}

Received: 10/04/2020; Revision: 12/04/2020; Accepted: 18/04/2020; Published: 20/04/2020

This work is licensed under a Creative Commons Attribution 4.0 International License.

\section{Abstract}

This continuing medical education (CME) outlines current national strategies to combat the pandemic of Corona virus (COVID 19). During a pandemic it is an obligation for each and every health care worker to be well versed with current guidelines. Three types of COVID dedicated health facilities as separate areas for suspect and confirmed cases are designed. Management of cases depend on their clinical status and result of COVID-19 testing. All three COVID dedicated health facilities of facilities are linked to the Surveillance team (IDSP). Isolation and quarantine play a key role in slowing the spread of COVID 19 and protecting people. Strict infection prevention and control practices are crucial in COVID 19 management. Evolving advisory on mask management, Chemoprophylaxis for COVID 19, Telemedicine in COVID 19, AYUSH in COVID 19, COVID-19 screening tool and others are to be carefully practiced. Ultimately prevention at individual, family, workplace and community level is the backbone of all strategies against COVID 19.

\section{Keywords}

\section{COVID-19; Patient; Health Care}

Corona viruses are family of viruses which may infect animals or humans. In humans, many coronaviruses exit ranging from the common cold to more severe diseases such as Middle East Respiratory Syndrome (MERS), Severe Acute Respiratory Syndrome (SARS) and others leading to respiratory illness.(1)] Currently global public health concern is potentially fatal disease, Coronavirus disease, is caused by SARSCOV2. A pneumonia of unknown cause detected in Wuhan; China was first reported to the WHO Country Office in China on 31 December 2019. A novel coronavirus ( $\mathrm{nCoV}$ ) is a new strain that has not been previously identified in humans. Study estimate that COVID-19 could be zoonotic origin as large number of infected people that were exposed to the wet animal market in Wuhan City, China. (2)

The outbreak was declared a Public Health Emergency of International Concern on 30 January 2020. On 11 February 2020, WHO announced a name for the new coronavirus disease as COVID-19. (2)

\section{Burden}

Globally, as of 9 April 2020, in humans there have been 1,356,780 confirmed cases of COVID-19, including 79,385 deaths, reported to WHO.(3) It has now spread to over 210 countries/territories, with reports of local transmission happening across the world. As of 15 April 2020 (17:00 GMT+5:30), according to Ministry of Health and family welfare, 
Government of India a total of 10197 COVID19 active

cases and 392 deaths, have been reported. (4)

Recent report on a Tiger being infected with the COVID 19 in New York is raising concerns possibilities of spread of the virus amongst animals also transmission of the virus from humans to animals and vice-versa. (5)

\section{Modes of transmission}

Person can catch COVID-19 from others who have the COVID-19 virus. The disease can spread from person to person during coughs or exhales via through small droplets from the nose or mouth. These droplets can land on objects and surfaces around the infected person. Other normal person gets COVID-19 by touching these contaminated objects or surfaces, then touching their eyes, nose or mouth. (1) The COVID-19 virus is found stable for several hours to days in aerosols and on surfaces, according to a new study from National Institutes of Health, CDC, UCLA and Princeton University scientists in The New England Journal of Medicine. COVID 19 virus is detectable in aerosols for up to three hours, up to four hours on copper, up to 24 hours on cardboard and up to two to three days on plastic and stainless steel. Hence people may acquire the COVID 19 virus through the air and after touching contaminated objects even after hours. (6) Droplet transmission of COVID 19 is reported to be prompted by air-conditioned ventilation.(7) Present data suggest COVID-19 virus is mainly transmitted through contact with respiratory droplets rather than through the air. Spread through faecal this route is not a main feature of the outbreak.

\section{Management of COVID cases Symptoms of COVID-19}

The most common symptoms of COVID-19 are fever, tiredness, and dry cough. Some patients may have aches and pains, nasal congestion, runny nose, sore throat or diarrhoea. The symptoms are usually mild and begin gradually. Some people become infected but are asymptomatic. (1) Elderly, and those with co morbidities like high blood pressure, heart problems or diabetes, are more likely to develop serious illness and difficulty breathing. Some studies had reported the children SARS-CoV-2, use the angiotensinconverting enzyme-2 (ACE2) as the cell receptor in humans. These are more common in adults than in children providing relative resistance to SARS-CoV-2 in children. However, there is lacunae of large sample paediatric COVID studies. (8)
Severe cases of COVID 19 can develop Multiple organ dysfunction syndrome. Available data in India suggests that nearly $70 \%$ of cases affected with COVID-19 either exhibit mild or very mild symptoms. Such cases may not require hospital admission. It is important to put in place mechanisms for triaging and decisions making for identification of the appropriate COVID dedicated facility for providing care to COVID-19 patients.

Quarantine and isolation are important mainstays of cluster containment. These measures help by breaking the chain of transmission. Quarantine refers to the separation of individuals who are not yet ill, but have been exposed to COVID 19 (i.e., have potential to become ill). There is voluntary home quarantine of contacts of suspect/confirmed cases, but they may also be quarantined in community based facilities. Isolation refers to separation of individuals who are ill and suspected or confirmed of COVID All suspect cases detected in the containment zones (till a diagnosis is made), will be hospitalized and kept in isolation till they test negative. Persons testing positive for COVID 19 will remain to be hospitalized till 2 of their samples test negative. (4)

COVID Patients to be categorized into three groups (1)

Group 1: Suspect and confirmed cases clinically assigned as mild and very mild (COVID Care Centres) Cases presenting with fever and/or upper respiratory tract illness (Influenza Like Illness). These patients will be accommodated in COVID Care Centers. The patients would be tested for COVID-19 and till such time their results are available they will remain in the "suspect cases" section of the COVID Care Center preferably in an individual room. Those who test positive, will be moved into the "confirmed cases" section of the COVID Care Center. If test results are negative, patient will be given symptomatic treatment and be discharged with advice to follow prescribed medications and preventive health measures

If any patient admitted to the COVID Care Center qualifies the clinical criteria for moderate

or severe case, such patient will be shifted respectively.

Group 2: Suspect and confirmed cases clinically assigned as moderate (Dedicated COVID Health Centres)

Pneumonia with no signs of severe disease (Respiratory Rate 15 to 30/minute, SpO2 90\%-94\%). Case will be admitted to Dedicated COVID Health 
centres. Those testing positives shall be shifted to "confirmed cases" section of Dedicated COVID Health Centre. Any patient, for whom the test results are negative, will be shifted to a non-COVID hospital and will be managed accordingly. Referral to Dedicated COVID Hospital as required.

Group 3: Suspect and confirmed cases clinically assigned as severe (Dedicated COVID Hospital)

Severe Pneumonia (with respiratory rate $\geq 30 /$ minute and/or SpO2 $<90 \%$ in room air) or ARDS or Septic shock. Such cases will be directly admitted to a Dedicated COVID Hospital's ICU till such time as test results are obtained. If test results are positive, such patient will remain in COVID-19 ICU and receive treatment as per standard treatment protocol. Patients testing negative to be managed with adequate infection prevention and control practices.

(1)

\section{Types of COVID Dedicated Facilities:}

\section{Fever Clinics}

In rural areas $\mathrm{CHCs}$, and in urban areas, the civil Igeneral hospitals, Urban CHCs and Municipal Hospitals can be designated as Fever Clinics. These could be set up preferably near the main entrance for triage and referral to appropriate COVID Dedicated Facility. Wherever space allows, a temporary make shift arrangement outside the facility may be arranged for this triaging. Referrals should be done as per the severity of patient.

\section{COVID Care Center (CCC):}

The COVID Care Centers offer care only for cases that have been clinically assigned as mild or very mild cases or COVID suspect cases. The COVID Care Centers are makeshift facilities. These may be set up in hostels, hotels, schools, stadiums, lodges etc., both public and private. If need be, existing quarantine facilities could also be converted into COVID Care Centers. Wherever a COVID Care Center is designated for admitting both the confirmed and the suspected cases, these facilities must have separate areas for suspected and confirmed cases with preferably separate entry and exit. Suspect and confirmed cases must not be allowed to mix under any circumstances. Preferably individual rooms should be assigned COVID Care Centers. Every Dedicated COVID Care Centre must necessarily be mapped to one or more Dedicated COVID Health Centres and at least one Dedicated COVID Hospital for referral purpose. Every Dedicated COVID Care Centre must also have a dedicated Basic Life Support Ambulance (BLSA) equipped with sufficient oxygen support on $24 \times 7$ basis, for ensuring safe referral. All three types of facilities will be linked to the Surveillance team. All facilities require to follow strict infection prevention and control practices

\section{Dedicated COVID Health Centre (DCHC):}

The Dedicated COVID Health Centre are hospitals that shall offer care for all cases that have been clinically assigned as moderate. These should either be a full private or government hospital or a separate block in a hospital with preferably separate entrylexit/zoning. Suspect and confirmed cases must not be allowed to mix under any circumstances. These hospitals would have beds with assured Oxygen support. Every Dedicated COVID Health Centre must necessarily be mapped to one or more Dedicated COVID Hospitals. Every Dedicated COVID Care Centre must also have a dedicated Basic Life Support Ambulance (BLSA) equipped with sufficient oxygen support on $24 \times 7$ basis, for ensuring safe referral.

\section{Dedicated COVID Hospital (DCH):}

The Dedicated COVID Hospitals are hospitals that shall offer comprehensive care primarily for those who have been clinically assigned as severe. The Dedicated COVID Hospitals should either be a full hospital or a separate block in a hospital with preferably separate entry lexit. Private hospitals may also be designated as COVID Dedicated Hospitals. These hospitals would have fully equipped ICUs, Ventilators and beds with assured Oxygen support. Suspect and confirmed cases should not be allowed to mix under any circumstances. They would also be referral centers for the Dedicated COVID Health Centers and the COVID Care Centers. (Figure 1)

\section{Laboratory diagnostics in COVID patient}

A suspected case, specimens is rapidly collected and tested. The Centers for Disease Control and Prevention (CDC) Interim Guidelines for Collecting, Handling, and Testing Clinical Specimens from Persons for Coronavirus Disease 2019 recommends collecting an upper respiratory specimen for initial diagnostic testing like Nasopharyngeal specimen (preferred), Oropharyngeal specimen, Nasal midturbinate specimen and Anterior nares specimen. Real-time reverse transcription polymerase chain reaction ( $r R T-P C R$ ) is the current gold standard for diagnosing suspected cases of COVID-19. rRT-PCR is a nucleic acid amplification test (NAAT) that detects unique sequences of the virus that causes COVID-19 (SARS-CoV-2) in respiratory tract specimens. Reverse Transcription Quantitative Polymerase Chain 
Reaction detect viral-specific antibodies (IgM and IgG) or antigens. (9) In addition another test required for the patient are shown in table 1 . In India COVID19 testing laboratory scale-up is fast tract. Presently across the country nearly 200 government and private hospital are testing for COVID 19. In response to the shortages of laboratory-based molecular testing capacity and reagents, the use of point-ofcare immunodiagnostic tests for COVID-19 is advised.

\section{Mask management}

Segregation of patients, healthcare workers and support staff as Covid-19 vs non-Covid-19 areas is vital during this pandemic. Proper use of N95 FFR (filtering facepiece respirator) /Triple layer surgical masks/linen masks is one of the crucial steps to reduce the risk of infection. Of N95 FFRs, requires training of all concerned staff, and dependent on compliance of all health care workers. Discrepancy in demand and supply chain led to advocacy of extended use of $\mathrm{N}-95$ masks to all health care workers involved all Non COVID Patient care areas for their own safety. Health care worker will be provided with five N-95 mask and brown cover. Each $\mathrm{N}-95$ mask to be placed in separate small paper bags as 1.2.3, and 4 and one is reserve. On day l, wear no. $1 \mathrm{~N} 95$ mask on duty. After duty used N-95 mask to be kept in bag 1 and let to dry out. Same to be done on day 2, dav 3 and day 4 . Reuse of day 1 N95 mask is recommended on day 5 . Such one cycle is for 20 days.(10)

\section{Chemoprophylaxis for COVID}

Hydroxy-chloroquine is found to be effective against coronavirus in laboratory studies and in vivo studies. Its use in prophylaxis is derived from available evidence of benefit as treatment and supported by pre-clinical data. The National Taskforce for COVID19 recommends the use of hydroxy-chloroquine for prophylaxis of SARS-CoV-2 infection for Asymptomatic healthcare workers involved in the care of suspected or confirmed cases of COVID-19: $400 \mathrm{mg}$ twice a day on Day 1, followed by $400 \mathrm{mg}$ once weekly for next 7 weeks, to be taken with meals and Asymptomatic household contacts Of laboratory confirmed cases: $400 \mathrm{mg}$ twice a day on Day 1,followed by $400 \mathrm{mg}$ once weekly for next 3 weeks; to be taken with meals. The drug is not recommended for prophylaxis in children under 15 years of age.(11)

\section{Imperative role of Telemedicine in COVID 19}

Currently when, when social distancing is among the major prevention measures, telemedicine services helps to access to quality healthcare to masses and prevent the transmission of COVID 19 by reducing the risks to both health care workers and patients and patients. It provides rapid access to health care workers who may not be available in person. This is a drastic change towards Digital Health in India. According to the Medical Council of India guidelines, doctors practicing telemedicine needs to complete a mandatory online course. However, present strategy enables hospitals to adapt to telehealth during a pandemic.(1)

\section{COVID Surveillance}

The containment zone are decided by the rapid response team based on the extent of cases/contacts listed and mapped. If contact listing/ mapping is taking time more than 12 hours), then arbitrary an area of $3 \mathrm{Kms}$ radius around the epicenter (the residence of the positive case) is containment zone. Containment zone can be refined based on the mapping of contacts and cases. A buffer zone is an additional $5 \mathrm{kms}$ radius $(7 \mathrm{Kms}$ in rural areas)/administrative

boundary of including neighboring districts/perurban zone.

Active Surveillance by each health worker would cover 50 houses in the sector assigned to them. The door to door surveillance are carried out under supervision of Medical Officers

within the defined surveillance zone. Passive Surveillance by all health facilities in the containment and buffer zones. All such facilities both in Government and Private sector (including clinic) reports clinically suspect cases of COVID-19.(12)

\section{Prevention}

\section{At Community level}

The lockdown restricts people from coming out of their homes. All transport services like road, air and rail are suspended with exceptions for transportation of essential goods, fire, police and emergency services. Educational institutions, industrial establishments and hospitality services are also suspended. Services such as food shops, banks and ATMs, petrol pumps, other essentials and their manufacturing are exempted. The anyone who fails to follow the restrictions can face up to penalty.

The COVID-19 pandemic is leading to social (physical) distancing policies worldwide, including in the USA. 
(13) Social distancing, also called "physical distancing," means keeping space between yourself and other people outside of your home. To practice social or physical distancing stay at least 1 to 2 meters away from other people. Stay out of crowded places and avoid mass gatherings. Social distancing is a best tool to slowing COVID spread locally and across the country and world. Social distancing also helps limit contact with infected people and contaminated surfaces. (14)

Make sure you, and the people around you, follow good respiratory hygiene. This means covering your mouth and nose with your bent elbow or tissue when you cough or sneeze. Then dispose of the used tissue immediately. A mask should be used by health workers, care takers, and individuals with respiratory symptoms, such as fever and cough. Mask community use depends on the availability of the logistics.

\section{At work place}

It is advisable workplaces should follow lock down. How essential workplace should be sanitized with sodium hypochlorite two hourly and whenever required. Everybody should use his personal soap and sanitizer. Employee should not do shake hands. Employee should not do touch your eyes. nose and mouth. Maintain personal hygiene and a safe distance (more than one meter) from persons during interaction. Cover nose and mouth with tissue or elbow while sneezing and coughing. Employee should not do participate in gatherings, including sitting in groups at canteens.

\section{At Family level}

Regularly check for the latest information on the COVID-19 available on the National, state and local public health authority website, helpline number, news, and others. If someone have a fever, cough and difficulty breathing, seek medical attention and call in advance. Follow the directions of your local health authority. If it is essential for to have someone bring you supplies or to go out, e.g. to buy food, then wear a mask to avoid infecting other people. Handle raw meat, milk or animal organs with care to avoid contamination of uncooked foods and avoid consuming raw or undercooked animal products. Practice regularly and thoroughly clean your hands for children.

Avoid large and small gatherings in private places and public spaces, such a friend's house, parks, restaurants, shops, or any other place. Children should not have in-person play dates while school is out. Work from home when possible. If possible, avoid using any kind of public transportation, ridesharing, or taxis. If you are a student or parent, talk to your school about options for digital/distance learning. Stay connected while staying away. It is very important to stay in touch with friends and family that don't live in your home. Call, video chat, or stay connected using social media. Everyone reacts differently to stressful situations and having to socially distance yourself from someone you love can be difficult.

\section{At Individual level}

Regularly and thoroughly clean your hands with an alcohol-based hand rub or wash them with soap and water. Maintain at least 2 metre (6 feet) distance between yourself and anyone who is coughing or sneezing. Avoid touching eyes, nose and mouth. Contaminated hands can transfer the virus to eyes, nose or mouth. Self-isolate by staying at home if one feel unwell, even with mild symptoms such as headache, low grade fever (37.3 C or above) and slight runny nose, until recovery.

\section{Addition for Health Care worker}

Proper use of personnel protective equipment's is crucial when Health care workers care for COVID patients. Knowledge of donning and doffing is essential for Health care workers. A study reported none of the 42,000 external healthcare workers who went to help Wuhan has been infected. Full protection with personnel protective equipment's, stringent protection and COVID-19 hospital level prevention and control management protocol, Patient management protocol and Cleaning and disinfection protocol.(15)

\section{Going back from Work place to home}

While going back home call one should call home when start from office. Someone at home should keep the front door open (don't touch the calling bell or door handle) and a bucket of water with washing soap powder or bleaching powder added to it in the front door. Keep things like car keys, pen, sanitizer bottle, phone in box outside the door. Wash your hands in the bucket and stand in the water for a few minutes. Meanwhile use tissue and sanitizer and wipe the items you have placed in the box. Wash your hands with soap water again. Now enter the house without touching anything. The bathroom door is kept open by someone and bucket of detergent soap water is ready. Take off all your clothes including innerwear and soak inside the bucket. Then take a head bath with a shampoo and 
body bath with soap. Wash your clothes put in washing machine with high temperature settings and dry clothes in direct sunlight.

\section{AYUSH in COVID 19}

While some western, traditional or home remedies may provide comfort and alleviate symptoms of COVID-19, there is no evidence that current medicine can prevent or cure the disease. Ministry of AYUSH recommends the following self-care guidelines for preventive health measures and boosting immunity with special reference to respiratory health. Drink warm water throughout the day. Daily practice of Yoga, Pranayama and meditation for at least 30 minutes. Spices like Turmeric, Cumin, Coriander and Garlic are recommended in cooking. Steam inhalation with fresh Mint leaves or Caraway seeds can be practiced once in a day. Clove powder mixed with natural sugar / honey can be taken 2-3 times a day in case of cough or throat irritation. (16)

\section{Initiatives}

\section{Pradhan Mantri Garib Kalyan Package}

It is an Insurance Scheme for Health Workers Fighting COVID 19. This accident insurance scheme covers Loss of life due to COVID19, and Accidental death on account of COVID-19 related duty. It covers Public healthcare providers including community health workers, who may have to be in direct contact and care of COVID-19 patients and who may be at risk of being impacted by this. Private hospital staff and retired /volunteer /local urban bodies/ contracted/daily wage /ad-hoc/outsourced staff requisitioned by States/ Central hospitals/ autonomous hospitals of Central/ States/UTs, AIIMS and INIs/ hospital of Central Ministries for COVID 19 related responsibilities. The duration of the policy is for a period of 90 days, starting from March 30, 2020. INR 50 LAKHS will be paid to the claimant of the insured person. (17)

\section{COVID-19 Screening Tool}

The Government of India had launched a mobile app developed in public-private partnership to bring the people of India together in a resolute fight against COVID-19. The App, called 'AarogyaSetu' joins Digital India for the health and well-being of every Indian. It enables people to assess themselves the risk for their catching the Corona Virus infection. It calculates this based on their interaction with others, using cutting edge Bluetooth technology, algorithms and artificial intelligence. (18) There are multiple other COVID screening applications freely available for self-risk assessment. (19)

\section{iGOT COVID}

Anticipating the exponential COVID case increase in future a larger force will be needed to replace the first line health care workers. For the training needs of the frontline workers, the launch of the iGOT platform has been fast-forwarded, and using the DIKSHA as a host. The platform will provide the training modules on flexi time and on-site basis so that the COVID response can be delivered at scale for the workforce needed to tackle the pandemic. (20)

\section{Conclusion}

India faces a significantly higher threat from COVID19 pandemic. In this article, we highlighted the updated management and prevention protocol for COVID 19 patients followed in India. A series of measures have been taken by both the Central and State Governments to break the chain of transmission. One among these is to isolate all suspect and confirmed cases of COVID-19. Symptoms, epidemiology, transmission, pathogenesis, and fatality directs COVID positive case , COVID suspect, contact, normal individual, families, communities and every one to play there role in combating this pandemic while system is working $24 / 7$ to analyse data, provide advice, coordinate with partners, preparedness, increase supplies and manage expert networks. Good policy, strategy and leadership, cooperation will be the key to slow the emerging COVID curve.

\section{Authors Contribution}

All authors have contributed equally.

\section{References}

1. Guidance document on appropriate management of suspect/confirmed cases of COVID-19. Ministry of Health \& Family Welfare, Directorate General of Health Services, EMR Division Available from https://www.mohfw.gov.in/pdf/FinalGuidanceonMa ngaementofCovidcasesversion2.pdf. [Last assessed on April 9, 2020]

2. Rothan HA, Byrareddy SN. The epidemiology and pathogenesis of coronavirus disease (COVID-19) outbreak [published online ahead of print, $2020 \mathrm{Feb}$ 26]. J Autoimmun. 2020;102433. doi:10.1016/j.jaut.2020.102433

3. Rolling updates on c1oronavirus disease (COVID-19), WHO, Available from https://www.who.int/emergencies/diseases/novelcoronavirus-2019/events-as-they-happen [Last assessed on April 9, 2020] 
4. COVID-19 INDIA. Available from https://www.mohfw.gov.in/[Last assessed on April 9, 2020]

5. Advisory regarding containing and management of COVID-19 in National Parks/Sanctuaries/Tiger Reserves. Ministry of Environment, Forest and Climate Change. Available from https://invest-indiarevamp-static-files.s3.ap-south-

1.amazonaws.com/s3fs-public/2020-

04/National\%20Park\%20tigers.pdf [Last assessed on April 8, 2020]

6. New coronavirus stable for hours on surfaces. Available from https://www.nih.gov/newsevents/news-releases/new-coronavirus-stablehours-surfaces [Last assessed on April 7, 2020]

7. Lu J, Gu J, Li K, Xu C, Su W, Lai Z, et al. COVID-19 outbreak associated with air conditioning in restaurant, Guangzhou, China, 2020. Emerg Infect Dis. 2020 Jul [9.4.2020]. https://doi.org/10.3201/eid2607.200764

8. Lee PI, Hu YL, Chen PY, Huang YC, Hsueh PR. Are children less susceptible to COVID-19? [published online ahead of print, 2020 Feb 25]. J Microbiol Immunol Infect. 2020;S1684-1182(20)30039-6. doi:10.1016/j.jmii.2020.02.011

9. IFCC Information Guide on COVID-19. Available from https://www.ifcc.org/ifcc-news/2020-03-26-ifccinformation-guide-on-covid-19/[Last assessed on April 7, 2020]

10. Standard operating procedure. (SOP's) for extended of $\mathrm{N}-95$ masks issued for personal safety of Health care Workers working in non Patient Care areas in AllMS hospital and various Centres. Available from https://www.aiims.edu/images/pdf/notice/Compreh ensive\%20Guideline_.pdf [Last assessed on April 15, 2020]

11. Advisory on the use of hydroxy-chloroquine prophylaxis for SARS-CoV-2 infection, Available from https://www.mohfw.gov.in/pdf/Advisoryontheuseof HydroxychloroquinasprophylaxisforSARSCoV2infecti on.pdf [Last assessed on April 5, 2020]

12. Micro Plan for Containing Local Transmission of Coronavirus Disease (COVID-19). Available from https://www.mohfw.gov.in/pdf/ModelMicroplanfor containmentoflocaltransmissionofCOVID19.pdf [Last assessed on April 15, 2020]
13. Bayham J, Fenichel EP. Impact of school closures for COVID-19 on the US health-care workforce and net mortality: a modelling study [published online ahead of print, $2020 \mathrm{Apr}$ 3]. Lancet Public Health. 2020;S2468-2667(20)30082-7. doi:10.1016/S24682667(20)30082-7

14. Social Distancing, Quarantine, and Isolation. Available from https://www.cdc.gov/coronavirus/2019ncov/prevent-getting-sick/social-distancing.html [Last assessed on April 1, 2020]

15. Analysis of 25,000 Lab-Confirmed COVID-19 Cases in Wuhan: Epidemiological Characteristics and NonPharmaceutical Intervention Effects. Available from https://cdn1.sph.harvard.edu/wpcontent/uploads/sites/21/2020/03/COVID-19-03-162020-Lin.pdf [Last assessed on April 5, 2020]

16. Ayurveda's immunity boosting measures for self care during COVID 19 crisis, Ministry of AYUSH . Available from https://invest-india-revamp-static-files.s3.apsouth-1.amazonaws.com/s3fs-public/202004/ImmunityBoostingAYUSHAdvisory.pdf[Last assessed on April 9, 2020]

17. Pradhan Mantri Garib Kalyan Package: Insurance Scheme for Health Workers Fighting COVID-19. Available from https://invest-india-revamp-staticfiles.s3.ap-south-1.amazonaws.com/s3fspublic/2020-

04/FAQPradhanMantriGaribKalyanPackagelnsurance SchemeforHealthWorkersFightingCOVID19.pdf [Last assessed on April 9, 2020]

18. AarogyaSetu: A multi-dimensional bridge. Available from https://invest-india-revamp-static-files.s3.apsouth-1.amazonaws.com/s3fs-public/2020-

04/Press\%20Information\%20Bureau_0.pdf [Last assessed on April 9, 2020]

19. 19. Available from https://www.apple.com/covid19 [Last assessed on April 9, 2020]

20. iGOT (Integrated Govt. Online Training) courses on DIKSHA platform on COVID-19 pandemic. Ministry of Personnel, Public Grievances \& Pensions. D/ o Personnel \& Training. Available from

21. https://invest-india-revamp-static-files.s3.ap-south1.amazonaws.com/s3fs-public/2020-

04/iGOTCOVID19Circular.pdf[Last assessed on April 9, 2020]

\section{Tables}

TABLE 1 SHOWING LABORATORY DIAGNOSTICS IN ADDITION TO IGM AND IGG IN COVID PATIENTS(9)

\begin{tabular}{|c|c|c|}
\hline Laboratory Test & $\begin{array}{l}\text { Main laboratory abnormalities observed } \\
\text { in adult patients with unfavorable } \\
\text { COVID-19 progression (Modified from 1- 12) }\end{array}$ & $\begin{array}{l}\text { Potential clinical and biological significance } \\
\text { (Modified from 3) }\end{array}$ \\
\hline \multirow{3}{*}{$\begin{array}{l}\text { Complete blood } \\
\text { count }\end{array}$} & Increased white blood cell & Bacterial (super)infection \\
\hline & Increase neutrophil count & Bacterial (super)infection \\
\hline & Decreased lymphocyte count & Decreased immunological response to the virus \\
\hline
\end{tabular}




\begin{tabular}{|c|c|c|}
\hline & Decreased platelet count & Consumption (disseminated) coagulopathy \\
\hline Albumin & Decreased & Impairment of liver function \\
\hline $\begin{array}{l}\text { Lactate } \\
\text { Dehydrogenase }\end{array}$ & Increased & $\begin{array}{l}\text { Pulmonary injury and/or widespread organ } \\
\text { damage }\end{array}$ \\
\hline $\begin{array}{l}\text { Alanine } \\
\text { Aminotransferase }\end{array}$ & Increased & Liver injury and/or widespread organ damage \\
\hline $\begin{array}{l}\text { Aspartate } \\
\text { aminotransferase }\end{array}$ & Increased & Liver injury and/or widespread organ damage \\
\hline Total bilirubin & Increased & Liver injury \\
\hline Creatinine & Increased & Kidney injury \\
\hline Cardiac troponin & Increased & Cardiac injury \\
\hline D-Dimer & Increased & $\begin{array}{l}\text { Activation of blood coagulation and/or } \\
\text { disseminated coagulopathy }\end{array}$ \\
\hline Prothrombin Time & Increased & $\begin{array}{l}\text { Activation of blood coagulation and/or } \\
\text { disseminated coagulopathy }\end{array}$ \\
\hline Procalcitonin & Increased & Bacterial (super)infection \\
\hline C-reactive protein & Increased & Severe viral infection/viremia/viral sepsis \\
\hline Ferritin & Increased & Severe inflammation \\
\hline Cytokines (IL-6) & Increased & Cytokine storm syndrome \\
\hline
\end{tabular}

\section{Figures}

FIGURE 1 FLOW DIAGRAM TO DEPICT FLOW OF COVID CASES AS PER SEVERITY (1)

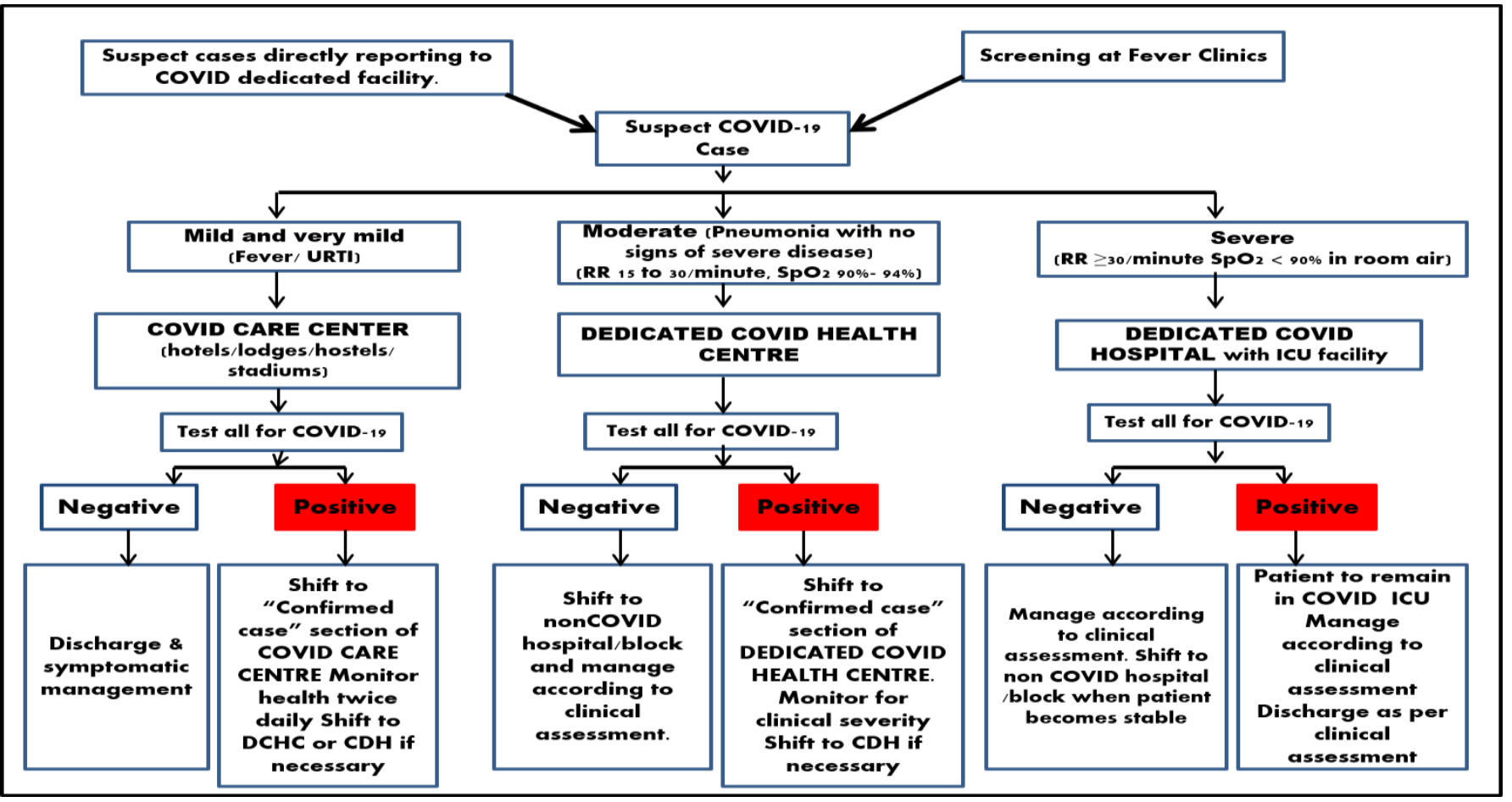

